\title{
FINITE RANK PERTURBATIONS OF SINGULAR SPECTRA
}

\author{
JAMES S. HOWLAND
}

\begin{abstract}
Let $T$ be selfadjoint, and $V$ nonnegative of finite rank, with the range of $V$ cyclic for $T$. Then the singular parts of $T$ and $H=T+V$ are supported on two sets $S_{T}$ and $S_{H}$ such that the multiplicity of $T$ on $S_{T} \cap S_{H}$ is less than the rank of $V$.
\end{abstract}

1. Introduction. In [3], Donoghue, following earlier work of Aronszajn, proved

1. THEOREM. Let $\phi$ be a cyclic vector for a selfadjoint operator $T$. For real $c \neq 0$, the singular parts of $T$ and $H=T+c\langle\cdot, \phi\rangle \phi$ are supported on disjoint sets.

To generalize this result to perturbations of rank higher than one is not completely straightforward, as a consideration of matrix examples will easily show. A certain generalization to nonnegative perturbations was given by the author in [4]. The criterion of that paper will be applied here to prove the following result:

2. THEOREM. Let $T$ be selfadjoint, $V$ a nonnegative operator of finite rank, and $H=T+V$. Assume that the range of $V$ is cyclie for $T$. Let $\mu_{T}$ and $n_{T}(\lambda)$ be a scalar spectral measure and multiplicity function of $T$, and define

$$
G=\left\{\lambda: n_{T}(\lambda)=\operatorname{rank} V\right\} .
$$

Then there exist sets $S_{T}$ and $S_{H}$ supporting the singular parts of $T$ and $H$ such that $S_{T} \cap G$ and $S_{H}$ are disjoint.

Note that $n_{T}(\lambda)$ cannot exceed the rank of $V$ when the range of $V$ is cyclic.

This has the following corollary, which is interesting even for matrices.

3. COROLlaRY. Let $T$ be selfadjoint, $V$ nonnegative of finite rank, and the range of $V$ cyclic for $T$. If $\lambda$ is an eigenvalue of $T$ with multiplicity equal to the rank of $V$, then $\lambda$ is not an eigenvalue of $H=T+V$.

For related work, see also $[\mathbf{1}, \mathbf{2}, \mathbf{5}]$.

2. Proofs. Note that the basic Hilbert space $\not$ is separable because the range $V \&$ of $V$ is cyclic. Multiplicity theory is therefore applicable.

For $\varepsilon>0$, define the function

$$
\delta_{\varepsilon}(t)=\frac{1}{\pi} \frac{\varepsilon}{t^{2}+\varepsilon^{2}} .
$$

As observed by Donoghue $[3, \S 1]$, the singular part of $T$ is supported by the measurable set

$$
S_{T}=\left\{\lambda: \lim _{\varepsilon \downarrow 0}\left\langle\delta_{\varepsilon}(T-\lambda) x, x\right\rangle=\infty \text { for some } x \in V \not\right\}
$$

and similarly for $H$.

Received by the editors March 4, 1985 and, in revised form, July 5, 1985.

1980 Mathematics Subject Classification. Primary 47A55.

${ }^{1}$ Supported by NSF Grant MCS-82-02115-01. 
Let $v_{1} \geq v_{2} \geq \cdots \geq v_{r}>0$ be the nonzero eignevalues of $V$, and $\phi_{1}, \ldots, \phi_{r}$ the corresponding normalized eigenvectors, which therefore are an orthonormal basis of $V H$. Because $V H$ is cyclic, one may choose

$$
\mu_{T}(S)=\sum_{j=1}^{r}\left\langle E_{T}[S] \phi_{j}, \phi_{j}\right\rangle
$$

as the scalar spectral measure, where $E_{T}$ is the spectral measure of $T$. Thus, $\left\langle E_{T}(d \lambda) \phi_{i}, \phi_{j}\right\rangle$ is absolutely continuous with respect to $\mu_{T}$. Define $M_{i j}(\lambda) \mu_{T}$-a.e. to be the Radon-Nikodým derivative

$$
\left\langle E_{T}(d \lambda) \phi_{i}, \phi_{j}\right\rangle=M_{i j}(\lambda) \mu_{T}(d \lambda)
$$

and $M(\lambda)$ to be the nonnegative matrix $M(\lambda)=\left\{M_{i j}(\lambda)\right\}_{i, j=1, \ldots, r}$. We shall regard $M(\lambda)$ as an operator on the space $V \mathscr{H}$. Let $m(\lambda)$ be the smallest eigenvalue of $M(\lambda)$ :

$$
m(\lambda)=\inf \left\{\langle M(\lambda) u, u\rangle:|u|^{2}=1, u \in V \mathcal{H}\right\},
$$

where $|u|$ is the norm of $u$ in $\nVdash$. Since $u$ may be restricted to a countable dense set, $m(\lambda)$ is measurable. One has

$$
M(\lambda) \geq m(\lambda) P
$$

where $P$ is the projection onto $V \nVdash$. Note also that

$$
m(\lambda) \leq 1 .
$$

Clearly, for all Borel sets $S,\left\langle E_{T}[S] \phi_{i}, \phi_{i}\right\rangle \leq \mu_{T}(S)$ so that $M_{i i}(\lambda) \leq 1 \mu_{T}$-a.e., and hence

$$
m(\lambda) \leq \min _{1 \leq i \leq r} M_{i i}(\lambda) \leq 1 .
$$

4. Lemma. One has

$$
n_{T}(\lambda)=\operatorname{rank} M(\lambda) \quad \mu_{T} \text {-a.e. }
$$

PROOF. This undoubtedly follows from the readers' favorite version of multiplicity theory. The author's favorite version is the Kato-Kuroda construction of direct integrals by spectral forms $[\mathbf{6}, \mathbf{7}]$. In that terminology, let $\mathcal{X}=V \mathcal{H}$, and

$$
f(\lambda, u)=\sum_{i j=1}^{r} u_{i} \bar{u}_{j} M_{i j}(\lambda) \equiv\langle M(\lambda) u, u\rangle
$$

for $u=u_{1} \phi_{1}+\cdots+u_{r} \phi_{r} \in \mathcal{X}$. Then $(f, \mathcal{X})$ is a spectral form for $T$ with respect to $\mu_{T}$, and the direct integral

$$
\mathscr{H} \cong \int_{\sigma(T)}^{\oplus} x(\lambda) \mu_{T}(d \lambda)
$$

diagonalizes $T$, where $X(\lambda)$ is the (completion of) the quotient space $X /\{u \in$ $\chi: f(\lambda, u)=0\}$. In this case, no completion is needed, since $\chi(\lambda)$ is the finitedimensional space $V \nVdash / \operatorname{ker} M(\lambda)$ whose dimension is $\operatorname{rank} M(\lambda)$.

The theorem of [4] will now be recalled. Let $K$ be another Hilbert space, and $A: \mathcal{K} \rightarrow \forall$ bounded. Let $T$ be selfadjoint, and assume that $A K$ is cyclic for $T$. 
5. PROPOSITION. The singular part of $H=T+A A^{*}$ is supported on the complement of the set of points $\lambda$ for which there is an $\eta>0$ such that

$$
A^{*} \delta_{\varepsilon}(T-\lambda) A \geq \eta I
$$

for all sufficiently small $\varepsilon>0$.

Note that $I$ in $(3)$ is the identity on $\mathcal{K}$, not $\mathcal{H}$. Note also that Proposition 5 implies Theorem 1 if one takes $K=\mathbf{C}$ (the complex numbers) and $A^{*}=c^{1 / 2}\langle\cdot, \phi\rangle$ (not $A$, as the misprint in [4] has it). Note finally that although $T$ was assumed bounded in [4], the proof there goes through unchanged for unbounded $T$.

To prove Theorem 2 , factor $V=A A^{*}$ through the space $K=V \mathscr{H}=V^{1 / 2} \not$ by defining $A: V^{1 / 2} \not / \not H \rightarrow \not$ as $A u=V^{1 / 2} u$. Then $A^{*}: \not \nvdash \rightarrow V^{1 / 2} \not /$ is also $A^{*} u=V^{1 / 2} u$, and $V=A A^{*}$. The identity $I$ in (3) is now the projection $P$ onto $V H$.

For fixed $\lambda$ and $u \in V \mathscr{H}$, one has

$$
\begin{aligned}
& \left\langle A^{*} \delta_{\varepsilon}(T-\lambda) A u, u\right\rangle=\left\langle\delta_{\varepsilon}(T-\lambda) V^{1 / 2} u, V^{1 / 2} u\right\rangle \\
& \quad=\sum_{i, j=1}^{r} u_{i} \bar{u}_{j} v_{i}^{1 / 2} v_{j}^{1 / 2}\left\langle\delta_{\varepsilon}(T-\lambda) \phi_{i}, \phi_{j}\right\rangle \\
& \quad \geq v_{r}\left\langle\delta_{\varepsilon}(T-\lambda) u, u\right\rangle=v_{r} \int \delta_{\varepsilon}(t-\lambda)\langle M(t) u, u\rangle \mu_{T}(d t) \\
& \geq v_{r}\|u\|^{2} \int \delta_{\varepsilon}(t-\lambda) m(t) \mu_{T}(d t)
\end{aligned}
$$

where (1) was used at the last step. Let $F$ be the set of all $\lambda$ for which

$$
\lim _{\varepsilon \downarrow 0} \int \delta_{\varepsilon}(t-\lambda) m(t) \mu_{T}(d t)=\infty .
$$

By (2), $F \subset S_{T}$, while by Proposition 5 , its complement $F^{c}$ supports the singular part of $H$. (In fact, by the proof in [5], $S_{H} \subset F^{c}$.)

Now $[3, \S 1] F$ supports the singular part of the measure $m(t) \mu_{T}(d t)$. The measure $\chi_{G}(t) \mu_{T}(d t)$ has the same null sets, because $G=\{t: m(t)>0\}$, and its singular part is supported by $S_{T} \cap G$. Thus $F$ and $S_{T} \cap G$ differ only by a set of $\mu_{T}$-measure zero. The result is then obtained by replacing $S_{T}$ by $S_{T}^{\prime}=S_{T} \sim\left(G \cap S_{T} \cap F^{c}\right)$.

\section{REFERENCES}

1. R. Bouldin, On the perturbation of the singular spectrum, Pacific J. Math. 34 (1970), 569-583.

2. R. W. Carey and J. D. Pincus, Unitary equivalence modulo the trace class for selfadjoint operators, Amer. J. Math. 98 (1976), 481-514.

3. W. F. Donoghue, On the perturbation of spectra, Comm. Pure Appl. Math. 18 (1965), 559-579.

4. J. S. Howland, On a theorem of Aronszajn and Donoghue on singular spectra, Duke Math J. 41 (1974), 141-143.

5. ( On the Kato-Rosemblum Theorem, Pacific J. Math. (to appear).

6. T. Kato and S. T. Kuroda, The abstract theory of scattering, Rocky Mountain J. Math. 1 (1971), 127-171.

7. _ Theory of simple scattering and eigenfunction expansions, Functional Analysis and Related Fields (F. Browder, ed.), Springer-Verlag, New York, 1970, pp. 99-131.

Department of Mathematics, UNiVersity of Virginia, ChaRlotTesville, ViRGINIA 22903 